\title{
Harmonic tori and generalised Jacobi varieties.
}

\author{
IAN MCINTOSH ${ }^{1}$
}

\section{Introduction.}

Over the last decade there has been considerable success in understanding the construction of certain harmonic 2-tori in symmetric spaces (in particular, the non-superminimal tori in $\mathbb{C P}^{n}$ and $S^{n}$ ) using the methods of integrable systems theory. For example, if $\varphi: M \rightarrow S^{2}$ is a non-conformal harmonic torus one knows that $\varphi$ is determined by its (real hyperelliptic) spectral curve $X$ equipped with a degree two function $\lambda$. There are already several ways of describing the relationship between the map $\varphi$ and its spectral data $(X, \lambda)$ : see, for example, $[2,7,12,10]$. But we lack a direct, geometric picture of how $\varphi$ arises from the algebraic geometry of $X$. What I want to present here is such a picture, based on a remarkable property of certain generalised Jacobi varieties. Moreover, it is quite straightforward to extend this picture to produce harmonic (indeed, pluri-harmonic) maps into Grassmannians and $\mathbb{P} U_{n+1}$ (i.e. $U_{n+1} /$ centre) and all of these will be maps of 'finite type' in the sense of [3]. From this picture one also sees that some of these harmonic tori are purely algebraic and these present an interesting class for further study.

To summarise, keeping with the example of non-conformal tori in $S^{2}$, let us suppose for simplicity that $X$ is non-singular with Jacobi variety $J$. One of the necessary properties of the pair $(X, \lambda)$ is that $\lambda$ is unbranched over $\lambda=1$ : let $O_{1}, O_{2}$ be the two points on $X$ with this value. Take $X^{\prime}$ to be the singularisation of $X$ obtained by identifying $O_{1}$ with $O_{2}$ to obtain an ordinary double point and let $J^{\prime}$ denote its generalised Jacobi variety. This is the moduli space of degree zero line bundles over $X^{\prime}$ : it is a non-trivial group extension of $J$ by the multiplicative complex numbers $\mathbb{C}^{\times}$. One may think of a line bundle over $X^{\prime}$ as a line bundle over $X$ with an identification between the fibres over $O_{1}$ and $O_{2}$ and it is this identification which is encoded by the fibres of $J^{\prime} \rightarrow J$. I claim that the harmonic map $\varphi$ factors

\footnotetext{
${ }^{1}$ Partially supported by NSF grants DMS-9626804 and DMS-9705479
} 
through $J^{\prime}$ i.e. $\varphi=\psi \circ \gamma$ where

$$
M \stackrel{\gamma}{\rightarrow} J^{\prime} \stackrel{\psi}{\rightarrow} \mathbb{P}^{1} .
$$

Moreover, the map $\psi$ is a rational map: it is the ratio of two sections of the pullback to $J^{\prime}$ of the $\theta$-line bundle over $J$. This pullback has an infinite dimensional space of sections but there is a natural spanning set indexed by $\mathbb{Z}$. The sections we want have index 0 and 1 . The map $\gamma$ is simply a homomorphism of real groups: we think of $M$ as $\mathbb{R}^{2}$ modulo a lattice, while $J^{\prime}$ has a real structure inherited from the real structure on $X$. The subgroup of real points $J_{R}^{\prime} \subset J^{\prime}$ is a compact real torus of dimension $g+1$ for $g=\operatorname{genus}(X)$. Indeed, if $z=x+i y$ denotes the standard complex coordinate on $\mathbb{R}^{2}$, then $\gamma$ is completely determined by the property that $\partial \gamma / \partial z$ is tangent to an Abel map image of $X^{\prime}$ in $J^{\prime}$. Although $\psi$ is only rational it is well-defined on the real slice $J_{R}^{\prime}$ of $J^{\prime}$ in which $\gamma$ takes its image, hence $\psi \circ \gamma$ is globally defined. Moreover, as we translate $\gamma(M)$ around $J_{R}^{\prime}$ we obtain new harmonic maps: this is a well-known property of maps of finite type. In fact we will see that a translation along the fibres of $J_{R}^{\prime} \rightarrow J_{R}$ merely changes the the image of $\varphi$ by an isometry.

The mechanism which underlies this construction is a beautiful fact about this generalised Jacobian. Given a line bundle $\mathcal{L}$ over $X$ of degree $g+1$ for which $\mathcal{L}\left(-O_{1}-O_{2}\right)$ is non-special one has, over a Zariski open subset of $J$, a rank two vector bundle $E$ whose fibre over $L \in J$ is $\Gamma(\mathcal{L} \otimes L)$, the space of globally holomorphic sections of $\mathcal{L} \otimes L$. In our case this open set contains the real subgroup $J_{R} \subset J$ and, locally, it is sections of $\mathbb{P} E$ (the projective bundle) over $J_{R}$ which provide the harmonic map (see [10]). However, when one tries to extend these sections globally one encounters non-trivial holonomy around the periods of $J$. It turns out that $J^{\prime}$ can be realised as a subbundle of the bundle of projective frames of $E$. The holonomy of these sections of $E$ takes values in the non-trival $\mathbb{C}^{\times}$-bundle $\pi: J^{\prime} \rightarrow J$. But $\pi^{*} J^{\prime}$ has a tautological section and this section is algebraic, hence the bundle of projective frames of $\pi^{*} E$ is algebraically trivialisable. Therefore the harmonic tori factor naturally through $J^{\prime}$.

Of course, to prove all this we need to have a working knowledge of generalised Jacobi varieties. Since I do not assume many differential geometers will be familiar with this the first two sections explain as much as we need to prove that $J^{\prime}$ is a natural subbundle of $\operatorname{PFr}(E)$, the bundle of projective frames of $E$ (indeed this is done for the whole Picard group Pic $\left(X^{\prime}\right)$ ). It follows that $\mathbb{P} F r\left(\pi^{*} E\right) \rightarrow J^{\prime}$ has a canonical trivialisation. This is necessary to turn sections of $G r_{k}\left(\pi^{*} E\right)$ (the bundle of $k$-planes) and $\operatorname{PFr}\left(\pi^{*} E\right)$ 
into maps from $J^{\prime}$ into $G r_{k, n+1}$ and $\mathbb{P} G l_{n+1}$. I also describe the appropriate Hermitian structure on fibres of $\pi^{*} E$ : this is necessary to be able to discuss real maps into the symmetric spaces $G r_{k, n+1}$ and $\mathbb{P} U_{n+1}$.

It is section 3 which shows that, for the right choice of sections restricted to the right choice of real two dimensional subgroup $\gamma\left(\mathbb{R}^{2}\right) \subset J^{\prime}$, one obtains harmonic maps. The most demanding part of the paper is to understand what constitutes 'the right choice'. Section 3 simply presents these choices as a fait accompli, but the motivation is as follows.

For simplicity let us return to the example of maps $\varphi: \mathbb{R}^{2} \rightarrow S^{2} \simeq \mathbb{C P}^{1}$. It is well-known (see [16]) that the harmonic maps are characterized by possessing a horizontal holomorphic lift into a loop group: we can represent this by the extended frame $F_{\lambda}$, which maps $\mathbb{R}^{2}$ into $C^{\omega}\left(\mathbb{C}^{\times}, G L_{2}\right)$ (recall that $\varphi$ is recovered by taking the complex line generated by the first column of $F_{\lambda}(z)$ evaluated at $\lambda=1$ ). We can think of the columns of $F_{\lambda}$ as being smooth maps of $\mathbb{R}^{2}$ into the space $\mathcal{H}$ of holomorphic sections of the trivial bundle $\mathbb{P}_{\lambda}^{1} \backslash\{0, \infty\} \times \mathbb{C}^{2}$. When $\varphi$ is doubly periodic it was shown in [10] that $\mathcal{H}$ is a rank 1 module over a commutative matrix algebra of symmetries of $F_{\lambda}^{-1} d F_{\lambda}$ (these are the polynomial Killing fields introduced by [3]). This algebra gives us the double cover $\lambda: X \rightarrow \mathbb{P}_{\lambda}^{1}$ and allows us to identify $\mathcal{H}$ with the space of holomorphic sections of a holomorphic line bundle $\mathcal{L}$ over $X \backslash \lambda^{-1}(\{0, \infty\})$. The columns of $F_{\lambda}(z)$ correspond to sections of $\mathcal{L}$. However, to extend these sections globally over $X$ we must make $\mathcal{L}$ move with $z$. The motion can be explicitly determined from a 1-cocycle on $X$ determined by the behaviour of $F_{\lambda}^{-1} \partial F_{\lambda} / \partial z$ about $\lambda=0, \infty$. One shows that $\mathcal{L}(z)$ moves linearly along $J$ and tangent to an Abel image of $X$ in $J$, in the manner described earlier. This gives us the right choice of subgroup $\gamma\left(\mathbb{R}^{2}\right) \subset$ $J^{\prime}$. The complex line generated by the first column of $F_{\lambda}(z)$ corresponds to a line in $\Gamma(\mathcal{L}(z)) \simeq \mathbb{C}^{2}$ determined purely by a divisor on $X$ supported over $\lambda=\infty$. The space $\mathbb{P} \Gamma(\mathcal{L}(z))$ is the fibre of $G r_{1}\left(\pi^{*} E\right)$ over $\mathcal{L}(z)$ and we see that the right choice of section of this bundle is determined by a divisor on $X$ over $\lambda=\infty$.

The same principle is known to apply to all the (non-isotropic) harmonic tori in $\mathbb{C P}^{n}[10,11]$. In section $3 \mathrm{I}$ have adapted this principle to the construction of harmonic maps into $G r_{k, n+1}$ and $\mathbb{P} U_{n+1}$. The first steps are to describe the divisor over $\lambda=0, \infty$, which picks out the right section of $G r_{k}\left(\pi^{*} E\right)$ (and later, $\mathbb{P} U\left(\pi^{*} E\right)$ ), and to describe the right direction to move along $J^{\prime}$. Then $F_{\lambda}$ is constructed and shown to frame a (pluri)-harmonic map. I fully expect that this construction gives all harmonic tori of semisimple finite type, in much the same way that one argues for $\mathbb{C P}^{n}[10]$. However, there is as yet no good description of the necessary and sufficient conditions 
for a torus in a symmetric space of rank greater than one to be of semisimple finite type, although some results have been obtained for $G r_{k, n+1}$ in [15].

The fourth section introduces the pullback of the $\theta$-line bundle to $J^{\prime}$ and shows that sections of this bundle provide maps $J^{\prime} \rightarrow G r_{k, n+1}$ (or $\left.\mathbb{P} U_{n+1}\right)$ which coincide with our harmonic maps on the appropriate subgroup $\gamma\left(\mathbb{R}^{2}\right) \subset J^{\prime}$. We now see that the harmonic map will be an algebraic object whenever this subgroup $\gamma\left(\mathbb{R}^{2}\right)$ is algebraic i.e. whenever it is the real slice of an algebraic subgroup of complex dimension two. I call these algebraic harmonic maps, although one must not be mislead: the (real) harmonic surface in $G r_{k, n+1}$ will not necessarily be real algebraic (since the map $J^{\prime} \rightarrow$ $G r$ won't in general be real) but it will be contained in a complex algebraic surface. It is easiest to discuss the case of maps into $\mathbb{C P}^{n}$ and here I show that if $\gamma\left(\mathbb{R}^{2}\right)$ is algebraic it is necessarily a two torus. Examples do exist: all $S^{1} \times S^{1}$-equivariant harmonic tori in $\mathbb{C P}^{n}$ are algebraic (and have the Riemann sphere as their spectral curve); the Gauss maps of the Delaunay surfaces are algebraic. But it remains an open and interesting question to find other examples.

Acknowledgements. This paper was written while the author was visiting the Center for Geometry, Analysis, Numerics and Graphics, Dept. of Mathematics, University of Massachusetts, Amherst.

\subsection{Spectral data.}

Throughout this article we will work with spectral data $(X, \lambda, \mathcal{L})$ where $X$ is a compact Riemann surface equipped with a rational function $\lambda$ of degree $n+1$ and a line bundle $\mathcal{L}$ over $X$ of degree $g+n$ where $g$ is the genus of $X$. The aim is that from this data one obtains harmonic maps into $G r_{k, n+1}$ or $\mathbb{P} U_{n+1}$ : certain extra properties of this data distinguish which target is obtained and whether or not the map is conformal. But in all cases $X$ must be equipped with real involution $\rho$ for which $\lambda \circ \rho=\bar{\lambda}^{-1}$, $\lambda$ must have no branch points on the unit circle and $\rho$ must fix all points over the pre-image of the this. It follows that the ramification divisor $R$ can be written as $R_{+}+\rho_{*} R_{+}$, where $R_{+}$is the divisor of ramification points lying over $|\lambda|<1$. Further, we insist that $\mathcal{L}$ satisfy a reality condition, namely, that the degree zero line bundle $L=\mathcal{L}\left(-R_{+}\right)$lie in the connected component of the identity, $J_{R}$, of the real subgroup $\left\{L \in J: \overline{\rho_{*} L} \cong L^{-1}\right\}$ of the Jacobian $J$ of $X$. Under these conditions one knows (cf. [10,11]) that $\operatorname{dim} \Gamma(\mathcal{L})=n+1$ and $\mathcal{L}$ has no non-zero global sections vanishing at all the $n+1$ distinct points $O_{1}, \ldots, O_{n+1}$ over $\lambda=1$. Moreover we can use 
the trace map $\operatorname{Tr}: \Gamma\left(\mathcal{O}_{X}(R)\right) \rightarrow \Gamma\left(\mathcal{O}_{\mathbb{P}^{1}}\right)$ to equip each space $\Gamma(\mathcal{L})$ with a Hermitian inner product. To fix this inner product we require, first, a global section $\sigma$ so that $\Gamma(\mathcal{L})$ can be realised as a divisor line bundle $\mathcal{O}_{X}(D)$, and second, an isomorphism $f$ of $\mathcal{O}_{X}\left(D+\rho_{*} D\right)$ with $\mathcal{O}_{X}(R)$ for which $\overline{\rho_{*} f}=f$ (we think of $f$ as a rational function with divisor $D+\rho_{*} D-R$ and it can be chosen to be positive over the unit circle $|\lambda|=1$, see [11]). Then the Hermitian inner product on $\Gamma(\mathcal{L})$ is given by

$$
\begin{aligned}
h\left(\sigma_{1}, \sigma_{2}\right) & =\operatorname{Tr}\left(f\left(\sigma_{1} \sigma^{-1}\right) \overline{\rho_{*}\left(\sigma_{2} \sigma^{-1}\right)}\right. \\
& =\sum_{j=1}^{n+1} f\left(O_{j}\right)\left(\sigma_{1} \sigma^{-1}\right)\left(O_{j}\right) \overline{\rho_{*}\left(\sigma_{2} \sigma^{-1}\right)\left(O_{j}\right)}
\end{aligned}
$$

Notice that if we change $\sigma$ then we must change $f$ too and as result we see that this Hermitian inner product is unique up to a real scaling (the scale of $f$ ).

We will use $J$ to denote the Jacobi variety of $X$ and $J^{\prime}$ to denote the generalised Jacobi variety for the singularisation $X^{\prime}$ of $X$ obtained by identifying all the points $O_{j}$ together to obtain a single node $O \in X^{\prime}$. Thus $J^{\prime}$ is the Jacobian for the modulus $0=O_{1}+\ldots+O_{n+1}$, in the terminology of Rosenlicht (see [13]). The next section describes the relevant properties of $J^{\prime}$.

Notation. If $E$ is a holomorphic vector bundle over a variety $M$ and $P \in M$ then $E_{P}$ will mean the stalk of the corresponding sheaf of locally holomorphic sections about $P$ while $E \mid P$ will mean the fibre of $E$ over $P$ (and similar notation for restriction to subvarieties of $M$ ).

\section{The generalised Jacobi variety.}

The aim in this section is to realise the generalised Jacobian $J^{\prime}$ of $X^{\prime}$ as a subbundle of the projective bundle of frames of a particular rank $n+1$ vector bundle over $J$.

Recall (from e.g. [13]) that $J^{\prime}$ is, by definition, the group of (isomorphism classes of) holomorphic line bundles of degree zero over $X^{\prime}$. Equally, it is the the group of equivalence classes of degree zero divisors on $X^{\prime}-\{O\}$ modulo the divisor 0 . Two such divisors $D_{1}, D_{2}$ are equivalent modulo o if $D_{1}-D_{2}$ is the divisor of a rational function $f \in \mathbb{C}(X)$ which takes the same non-zero value at each point $O_{j}$. We will denote the divisor class of $D \bmod o$ by $[D]_{\circ}$ and the divisor of any rational function $f$ by $(f)$. 
Since this is a refinement of the divisor classes on $X$ the normalisation $X \rightarrow X^{\prime}$ induces, by pullback, the extension

$$
1 \rightarrow H_{\mathfrak{o}} \rightarrow J^{\prime} \stackrel{\pi}{\rightarrow} J \rightarrow 1
$$

where $H_{0}=\left\{[D]_{0}: D\right.$ is the divisor of a rational function on $\left.X\right\}$. For our purposes it is more useful to describe $H_{0}$ as follows. Let $U_{\mathfrak{o}}$ be the multiplicative group of rational functions on $X$ which have no poles or zeroes on $\mathcal{o}$ and let $U_{O}$ denote the subgroup of those which are obtained by pullback from $X^{\prime}$ (all such functions have $f\left(O_{j}\right)=f\left(O_{1}\right)$ for each point $O_{j}$ ). Then $U_{0} / U_{O}$ is isomorphic to $H_{0}$ : the identification gives to each $f \in U_{0}$ its divisor class mod 0 . This allows a local description of the fibres of $J^{\prime}$ over $J$. For each $L \in J$ let $L_{0}$ be the space of locally regular sections of $L$ about the support of $\mathfrak{o}$ and let $L_{\mathfrak{o}}^{\times}$be the subset of those which do not vanish at any $O_{j}$. Then $U_{O}$ acts by multiplication on $L_{0}^{x}$ and

$$
J^{\prime} \mid L \simeq L_{\mathfrak{o}}^{\times} / U_{O} .
$$

This equally well describes the fibres of the Picard variety extension $\pi$ : $\operatorname{Pic}\left(X^{\prime}\right) \rightarrow \operatorname{Pic}(X)$ where these are the groups of line bundles of any degree. From another point of view, the fibre over $L \in P i c(X)$ consists of all n-tuples $\left(g_{2}, \ldots, g_{n+1}\right)$ of linear bijections $g_{j}: L\left|O_{j} \rightarrow L\right| O_{1}$.

Now we want to realise $P i c\left(X^{\prime}\right)$ as a subbundle of a natural frame bundle over $\operatorname{Pic}(X)$. Let $\mathcal{E} \rightarrow X \times \operatorname{Pic}(X)$ be a Poincare line bundle i.e. a line bundle with the property $\mathcal{E} \mid X \times\{L\} \simeq L$. Recall (from e.g. [1]) that $\mathcal{E}$ is not unique: if $\mathcal{T} \rightarrow \operatorname{Pic}(X)$ is any line bundle and $\pi_{2}: X \times \operatorname{Pic}(X) \rightarrow \operatorname{Pic}(X)$ is projection on the second factor then $\mathcal{E} \otimes \pi_{2}^{*} \mathcal{T}$ is also a Poincare line bundle. The normalisation $X \rightarrow X^{\prime}$ pushes $\mathcal{E}$ down to a coherent sheaf whose restriction to $\{O\} \times \operatorname{Pic}(X)$ we will call $E$. Its fibre over any point $L$ is

$$
E\left|L \simeq L_{\mathfrak{o}} /\left(\mathfrak{m}_{O} \cdot L_{\mathfrak{o}}\right)=\oplus_{j=1}^{n+1} L\right| O_{j}
$$

where $\mathfrak{m}_{O}$ is the ideal in $\mathcal{O}_{\mathfrak{o}}$ of locally regular functions about $\mathfrak{o}$ with divisor of zeroes at least $\boldsymbol{o}$. Thus $E$ is a rank $n+1$ vector bundle. Let $\mathbb{P} F r(E)$ be the principal bundle of projective frames of $E$ and let $G r_{k}\left(\pi^{*} E\right)$ be the Grassmannian bundle of $k$-planes in the fibres of $\pi^{*} E$. Since the effect of tensoring $\mathcal{E}$ by $\pi_{2}^{*} \mathcal{T}$ is to globally rescale each line bundle $L$, these projective bundles are independent of the choice of Poincare line bundle.

Proposition 1. There exists a canonical injective morphism of principal bundles $\operatorname{Pic}\left(X^{\prime}\right) \rightarrow \mathbb{P F r}(E)$. Consequently $\mathbb{P} F r\left(\pi^{*} E\right)$ and $G r_{k}\left(\pi^{*} E\right)$ are canonically trivial. 
Proof. Set $G^{\mathbb{C}}=\mathbb{P} G L_{n+1}$ and let $H \subset G^{\mathbb{C}}$ be the subgroup of diagonal matrices modulo scaling. Consider $\mathbb{P} F r(E)$ as a principal right $G^{\mathbb{C}}$-bundle over $J$ in the usual way. In particular, for $f=\left[f_{1}, \ldots, f_{n}\right]$ a projective frame and $h=\left[\operatorname{diag}\left(\mathrm{h}_{\mathrm{j}}\right)\right] \in \mathrm{H}$ this means

$$
f h=\left[h_{1}^{-1} f_{1}, \ldots, h_{n}^{-1} f_{n}\right] .
$$

Also consider $J^{\prime}$ as a principal $H$-bundle by identifying $H$ with $H_{\mathfrak{o}}$ via the epimorphism

$$
U_{\mathrm{o}} \rightarrow H, \quad f \mapsto\left[\operatorname{diag}\left(\mathrm{f}\left(\mathrm{O}_{\mathrm{j}}\right)\right)\right],
$$

which has kernel $U_{O}$. We will construct a principal bundle morphism $\mu$ : $J^{\prime} \rightarrow \mathbb{P} F r(E)$ such that $\mu\left(h^{-1} x\right)=\mu(x) h$ for $x \in J^{\prime}$ and $h \in H$. By (2.3), for each $L \in \operatorname{Pic}(X)$ we have a natural map $L_{0}^{\times} \rightarrow \operatorname{Fr}(E) \mid L$ which assigns to a local section $\sigma$ its restrictions $\left(\sigma\left|O_{1}, \ldots, \sigma\right| O_{n+1}\right)$. Now if $f \in U_{O}$ then $f \sigma$ is mapped to $\left(c \sigma\left|O_{1}, \ldots, c \sigma\right| O_{n+1}\right)$ where $c$ is the value $f$ takes at every $O_{j}$. This induces a injection

$$
\mu: J^{\prime}\left|L \simeq L_{\mathrm{o}}^{\times} / U_{O} \rightarrow \mathbb{P} F r(E)\right| L ; \quad[\sigma] \mapsto\left[\sigma\left|O_{1}, \ldots, \sigma\right| O_{n+1}\right]
$$

where square brackets denote the respective equivalence classes. This map is clearly $H$-equivariant. By globalising this we obtain an injective morphism of principal bundles.

Now, $\pi^{*} \operatorname{Pic}\left(X^{\prime}\right)$ is trivialisable by its tautological section (and a little thought shows this must actually be an algebraic section), whence $\operatorname{PFr}\left(\pi^{*} E\right)$ is canonically trivialisable and so are all the bundles $G r_{k}\left(\pi^{*} E\right)$.

It is an easy exercise to verify that, at each point $L^{\prime} \in P i c\left(X^{\prime}\right)$ with $\pi\left(L^{\prime}\right)=L$, this canonical trivialisation maps

$$
\mathbb{P} \pi^{*} E \mid L^{\prime} \rightarrow \mathbb{P}^{n}, \quad\left[\sum v_{j} s_{j}\right] \mapsto\left[v_{1}, \ldots, v_{n}\right],
$$

where $s_{j} \in L \mid O_{j}$ are such that $L^{\prime}$ is obtained from $L$ by identifying fibres so that $s_{j}$ is identified with $s_{1}$.

For later use, let us now show that any section of either $\operatorname{PFr}\left(\pi^{*} E\right)$ or $G r_{k}\left(\pi^{*} E\right)$ which is lifted from a section of $\mathbb{P F r}(E)$ or $G r_{k}(E)$ produces, by the trivialisation, an $H$-equivariant map of $J^{\prime}$ into $G^{\mathbb{C}}$ or $G r_{k, n+1}$. To show this we need to examine more closely the canonical trivialisation. Let $\chi: J^{\prime} \rightarrow \pi^{*} J^{\prime}$ be the tautological section and lift $\mu$ to $\mu^{\prime}: \pi^{*} J^{\prime} \rightarrow \mathbb{P} F r\left(\pi^{*} E\right)$. Then we have a section

$$
\tau=\mu^{\prime} \circ \chi: J^{\prime} \rightarrow \mathbb{P} F r\left(\pi^{*} E\right)
$$


with the property $\tau(h x)=\tau(x) h^{-1}$ for $h \in H, x \in J^{\prime}$. This induces the canonical trivialisation

$$
\operatorname{PFr}\left(\pi^{*} E\right) \rightarrow J^{\prime} \times G^{\mathbb{C}} ; \quad \tau(x) g \mapsto(x, g) .
$$

Lemma 1. Let $\sigma^{\prime}$ be a section of $\mathbb{P} F r\left(\pi^{*} E\right)$ and $\psi: J^{\prime} \rightarrow G^{\mathbb{C}}$ be the map it defines by $\sigma^{\prime}(x)=\tau(x) \psi(x)$. Then $\psi(h x)=h \psi(x)$ for all $h \in H$ if and only if $\sigma^{\prime}$ is the lift of a section $\sigma$ of $\mathbb{P} F r(E)$ over $J$.

Proof. Suppose $\sigma^{\prime}$ is such a lift, then $\sigma^{\prime}(h x)=\sigma^{\prime}$ for $h \in H$. But

$$
\sigma^{\prime}(h x)=\tau(h x) \psi(h x)=\tau(x) h^{-1} \psi(h x)
$$

whence $h^{-1} \psi(h x)=\psi(x)$. Clearly the identities reverse to give us the converse.

Notice that the lemma remains true if we replace $\mathbb{P} F r(E)$ by $G r_{k}(E)$ and $G^{\mathbb{C}}$ by $G r_{k, n+1}$. In this case we identify $G r_{k}\left(\pi^{*} E\right)$ with $\mathbb{P F r}\left(\pi^{*} E\right) \times_{G^{\mathbb{C}}}$ $G r_{k, n+1}$ so that its canonical trivialisation can be written

$$
\mathbb{P} F r\left(\pi^{*} E\right) \times{ }_{G^{\mathrm{C}}} G r_{k, n+1} \rightarrow J^{\prime} \times G r_{k, n+1} ; \quad[\tau(x) g, V] \mapsto(x, g V) g \in G^{\mathbb{C}} .
$$

Again, square brackets denote an equivalence class. Thus a section $\sigma^{\prime}$ of $G r_{k}\left(\pi^{*} E\right)$ defines a map $\psi: J^{\prime} \rightarrow G r_{k, n+1}$ by $\sigma^{\prime}(x)=[\tau(x), \psi(x)]$.

\subsection{Hermitian structure.}

So far we have not used the reality conditions on $(X, \lambda, \mathcal{L})$ at all: indeed, all we required were $n+1$ distinct points on $X$. The reality conditions arise because we must view $G r_{k, n+1}$ and $\mathbb{P} U_{n+1}$ as symmetric spaces. Each of these has, up to scaling, a unique group invariant metric. Because the maps we construct will be equi-harmonic (i.e. harmonic with respect to any of these group invariant metrics) the scaling will prove irrelevant. But the construction we are going to use, from [10], works directly with $\Gamma(\mathcal{L})$ equipped with the inner product (1.1). So the aim of this section is to show how this fits in with the fibres on $\pi^{*} E$ and the inner product they obtain from the projective trivialisation.

First, we must restrict our attention to a particular real submanifold of $\operatorname{Pic}\left(X^{\prime}\right)$. Define $J_{R}^{\prime}$ to be the connected component of the identity of

$$
\left\{L^{\prime} \in J^{\prime}: L^{\prime-1} \simeq \overline{\rho_{*} L^{\prime}}\right\}
$$


and set

$$
N^{\prime}=\left\{L^{\prime}\left(R_{+}\right): L^{\prime} \in J_{R}^{\prime}\right\},
$$

where we recall $R_{+}$is the divisor of those ramification points lying over $|\lambda|<1$. Notice that $N^{\prime}$ consists of bundles of degree $g+n$. Now for each $\mathcal{L}^{\prime} \in N^{\prime}$ with $\mathcal{L}=\pi\left(\mathcal{L}^{\prime}\right)$ we have vector space isomorphisms

$$
\Gamma(\mathcal{L}) \rightarrow \bigoplus_{j=1}^{n+1} \mathcal{L} \mid O_{j} \rightarrow\left(\mathcal{L} \mid O_{1}\right)^{n+1}
$$

where the first arrow is restriction to the fibres: this is bijective because (we recall from section 1.1) $\mathcal{L}$ has no non-zero global sections vanishing at all the points $O_{j}$ and $\operatorname{dim} \Gamma(\mathcal{L})=n+1$. The second arrow is the identification of fibres $\mathcal{L}\left|O_{j} \rightarrow \mathcal{L}\right| O_{1}$ encoded in $\mathcal{L}^{\prime}$. Indeed, this is how the canonical trivialisation of $\mathbb{P} \pi^{*} E$ works. Notice that $\Gamma\left(\mathcal{L}^{\prime}\right)$ is one dimensional since it consists of all global sections of $\mathcal{L}$ satisfying the $n$ fibre identifications. Therefore, given $\mathcal{L}^{\prime} \in N^{\prime}$, we can equip $\Gamma(\mathcal{L})$ with another Hermitian inner product, unique up to positive real scaling, given by the formula

$$
\tilde{h}\left(\sigma_{1}, \sigma_{2}\right)=\sum_{j=1}^{n+1}\left(\frac{\sigma_{1} \mid O_{j}}{\sigma \mid O_{j}}\right) \overline{\left(\frac{\sigma_{2} \mid O_{j}}{\sigma \mid O_{j}}\right)}
$$

where $\sigma \in \Gamma\left(\mathcal{L}^{\prime}\right)$ is non-zero. It is this inner product which the canonical trivialisation of $\pi^{*} E$ induces. The next lemma shows that provided we work with $\pi^{*} E \mid N^{\prime}$ the two Hermitian structures agree.

Lemma 2. Let $\mathcal{L}^{\prime} \in N^{\prime}$ and set $\mathcal{L}=\pi\left(\mathcal{L}^{\prime}\right)$. Then the two Hermitian inner products $h$ and $\tilde{h}$ on $\Gamma(\mathcal{L})$, given by (1.1) and (2.5) respectively, agree (up to real scaling).

Proof. Since $\mathcal{L}^{\prime}$ has a non-zero global section, $\mathcal{L}^{\prime} \simeq \mathcal{O}_{X^{\prime}}(D)$ where $D$ is a positive divisor of degree $g+n$. This divisor $D$ is unique since $\operatorname{dim} \Gamma\left(\mathcal{L}^{\prime}\right)=1$. By definition of $N^{\prime}, D+\rho_{*} D-R$ is the divisor of a rational function $f$ taking the same value at each point $O_{j}$. Indeed, we may choose $f$ so that $\overline{\rho_{*} f}=f$ and we may assume that $f$ is positive over $|\lambda|=1$ ([11, lemma 2]). Let $\sigma \in \Gamma\left(\mathcal{L}^{\prime}\right) \subset \Gamma(\mathcal{L})$ be a non-zero section, then $\sigma$ has divisor $D$ so on $\Gamma(\mathcal{L})$ we have

$$
h\left(\sigma_{1}, \sigma_{2}\right)=\sum_{j=1}^{n+1} f\left(O_{j}\right)\left(\sigma_{1} \sigma^{-1}\right)\left(O_{j}\right) \overline{\rho_{*}\left(\sigma_{2} \sigma^{-1}\right)\left(O_{j}\right)}=f\left(O_{1}\right) \tilde{h}\left(\sigma_{1}, \sigma_{2}\right),
$$


and $f\left(O_{1}\right)$ is real and positive.

From now on we will work exclusively with the bundle $E^{\prime}=\pi^{*} E \mid N^{\prime}$. Let $\tau: N^{\prime} \rightarrow \mathbb{P} F r\left(E^{\prime}\right)$ be its canonical projective framing and let $\mathbb{P} U\left(E^{\prime}\right)$ be the pullback of $N^{\prime} \times \mathbb{P} U_{n+1}$ by the trivialisation of $\mathbb{P} F r\left(E^{\prime}\right)$ induced by $\tau$. Of course, this makes $\tau$ a section of $\mathbb{P} U\left(E^{\prime}\right)$. This is a bundle of projective unitary groups and induces a Hermitian symmetric space structure on each fibre of $G r_{k}\left(E^{\prime}\right)$. From the definition of $\tilde{h}$ and the previous lemma we see that this structure is projectively equivalent with that induced by the fibrewise Hermitian metric $h$ on $E^{\prime}$. It follows that the inclusion $J_{R}^{\prime} \hookrightarrow \mathbb{P} U\left(E^{\prime}\right)$ embeds $J_{R}^{\prime} \cap H_{\mathrm{o}}$ into $\mathbb{P} U_{n+1}$, hence $J_{R}^{\prime}$ is a real torus of dimension $g+n$.

\section{Harmonic maps into $G r_{k, n+1}$ and $\mathbb{P} U_{n+1}$.}

For simplicity, set $G=\mathbb{P} U_{n+1}$. We need to recall the characterisation of (equi)-harmonic maps into $G r_{k, n+1}$ and $\mathbb{P} U_{n+1}$ using maps into the loop group $\Lambda G$ of real-analytic maps $S^{1} \rightarrow G$ (cf. $[3,16]$ ). Recall that $G r_{k, n+1}$ is isomorphic to the homogeneous space $G / K$ where $K$ is the fixed point subgroup of the involution $g \mapsto \nu g \nu^{-1}$ determined by

$$
\nu=\left(\begin{array}{cc}
I_{k} & 0 \\
0 & -I_{n+1-k}
\end{array}\right)
$$

where $I_{k}$ is the $k \times k$ identity matrix. This involution induces a symmetric decomposition of $\mathfrak{g}$, the Lie algebra of $G$, into $\mathfrak{g}=\mathfrak{k}+\mathfrak{m}$, where $\mathfrak{k}$ is the Lie algebra for $K$. Recall (from e.g. [4]) that a (based) map

$$
\varphi: \mathbb{R}^{2} \rightarrow G / K, \quad f(0)=1 \cdot K
$$

is harmonic iff it possesses an extended frame $F_{\zeta}: \mathbb{R}^{2} \rightarrow \Lambda G$ (i.e. $F_{1}$ frames $\varphi)$ for which

$$
F_{\zeta}^{-1} \partial F_{\zeta} / \partial z=\zeta^{-1} A_{-1}+A_{0}
$$

where $z=x+i y, A_{0}$ takes values in $\mathfrak{k}^{\mathbb{C}}(=\mathfrak{k} \otimes \mathbb{C})$ and $A_{-1}$ takes values in $\mathfrak{m}^{\mathbb{C}}$. Now let $\mathfrak{m}_{-}^{\mathbb{C}}$ and $\mathfrak{m}_{+}^{\mathbb{C}}$ denote the subspaces of, respectively, lower triangular and upper triangular matrices in $\mathfrak{m}^{\mathbb{C}}$. Then a useful corollary to the above fact is:

Lemma 3. Let $F_{\lambda}: \mathbb{R}^{2} \rightarrow \Lambda G$ satisfy

$$
F_{\lambda}^{-1} \partial F_{\lambda} / \partial z=\lambda^{-1} a_{-1}+a_{0}
$$


where $a_{0}$ takes values in $\mathfrak{k}^{\mathbb{C}}+\mathfrak{m}_{+}^{\mathbb{C}}$ and $a_{-1}$ takes values in $\mathfrak{m}_{-}^{\mathbb{C}}$. Then $F_{1}$ frames a harmonic map into $G r_{k, n+1}$.

Proof. Define

$$
\kappa=\left(\begin{array}{cc}
I_{k} & 0 \\
0 & \zeta I_{n+1-k}
\end{array}\right)
$$

where $\zeta=\sqrt{\lambda}$. One easily checks that $\tilde{F}_{\zeta}=\kappa^{-1} F_{\zeta^{2}} \kappa$ satisfies (3.1) and therefore $F_{1}=\tilde{F}_{1}$ frames a harmonic map into $G r_{k, n+1}$.

We will also need the analogous characterisation of harmonic maps $\varphi$ : $\mathbb{R}^{2} \rightarrow G$. Recall from [16] that $\varphi$ is harmonic if and only if it possesses an extended frame $F_{\lambda}: \mathbb{R}^{2} \rightarrow \Lambda G$ for which

$$
F_{\lambda}^{-1} \partial F_{\lambda} / \partial z=\left(1-\lambda^{-1}\right) A
$$

where $A$ takes values in $\mathfrak{g}^{\mathbb{C}}$.

\subsection{Maps into $G r_{k, n+1}$.}

As explained in the introduction, when we choose the right section of $\operatorname{Gr}\left(E^{\prime}\right)$ restricted to the right directions along $J^{\prime}$ we will obtain harmonic maps. These choices are made as follows.

We assume, without loss of generality, that $k \leq(n+1) / 2$. Let $(X, \lambda, \mathcal{L})$ be spectral data as described above and let us further insist that $\lambda$ has at least $k$ zeroes $P_{1}, \ldots, P_{k}$ of order two and therefore $k$ poles $Q_{1}, \ldots, Q_{k}$ of order two given by $Q_{j}=\rho\left(P_{j}\right)$. Define the positive divisor $D_{\infty}$ of degree $n+1-k$ by

$$
D_{\infty}=(\lambda)_{\infty}-Q_{1}-\ldots-Q_{k},
$$

where $(\lambda)_{\infty}$ is the divisor of poles of $\lambda$. By earlier remarks, $\mathcal{L}\left(-(\lambda)_{\infty}\right)$ is nonspecial and it follows by Riemann-Roch that $\Gamma\left(\mathcal{L}\left(-D_{\infty}\right)\right)$ has dimension $k$. So $D_{\infty}$ determines a rank $k$ subbundle of the vector bundle $E^{\prime}$ and therefore a section of $G r_{k}\left(E^{\prime}\right)$. By applying the canonical trivialisation we obtain a map $N^{\prime} \rightarrow G r_{k, n+1}$. For each $\mathcal{L}^{\prime} \in N^{\prime}$ we have an isomorphism $J_{R}^{\prime} \rightarrow N^{\prime}$ and the combination of these two maps we will define to be

$$
\psi_{\mathcal{L}^{\prime}}: J_{R}^{\prime} \rightarrow G r_{k, n+1} \text {. }
$$


Notice that the spectral data only determines this map up to the choice of $\mathcal{L}^{\prime}$ over $\mathcal{L}$. However, the group $H_{\mathfrak{o}} \cap J_{R}^{\prime}$ acts transitively on each fibre of $\pi$ : $N^{\prime} \rightarrow N$ and this action induces isometries after canonical trivialisation of $\operatorname{PFr}\left(E^{\prime}\right)$, so the map $\psi_{\mathcal{L}^{\prime}}$ is determined by the spectral data up to isometries of the target.

Now we will define the real homomorphism $\gamma: \mathbb{R}^{2 k} \rightarrow J_{R}^{\prime}$ for which $\varphi=\psi_{\mathcal{L}^{\prime}} \circ \gamma$ is pluri-harmonic. Set $X_{o}^{\prime}=X^{\prime}-\{O\}$ and recall (from e.g. [13]) that there is, for any point $B \in X_{o}^{\prime}$, an Abel map

$$
\mathcal{A}_{B}^{\prime}: X_{o}^{\prime} \rightarrow J^{\prime} \simeq \Gamma\left(\Omega_{X^{\prime}}\right)^{*} / H_{1}\left(X_{o}^{\prime}, \mathbb{Z}\right), \quad P \mapsto \int_{B}^{P}
$$

with the integral considered modulo periods. About each $P_{j}$ we have a local coordinate $\zeta_{j}=\sqrt{\lambda}$. We characterise $\gamma$ by

$$
\partial \gamma /\left.\partial z_{j}\right|_{z=0}=\partial \mathcal{A}_{P_{j}}^{\prime} /\left.\partial \zeta_{j}\right|_{\zeta_{j}=0}
$$

where $z=\left(z_{1}, \ldots, z_{k}\right)$ are the standard complex coordinates on $\mathbb{R}^{2 k}$.

Theorem 1. For each $\mathcal{L}^{\prime} \in N^{\prime}$ the map $\varphi=\psi_{\mathcal{L}^{\prime}} \circ \gamma: \mathbb{R}^{2 k} \rightarrow G r_{k, n+1}$ is pluri-harmonic. Moreover, $\varphi\left(z_{m}\right)$ is non-conformal for each $m=1, \ldots, k$.

Before presenting the proof we need a few preliminary results.

Lemma 4. The real homomorphism $\gamma: \mathbb{R}^{2 k} \rightarrow J_{R}^{\prime}$ is an immersion (except when $2 k=g+n+1$, which can only happen for $g=0$ ).

Proof. Set $D=P_{1}+\ldots+P_{k}$ and notice that $\gamma$ is tangent at the identity to $\mathcal{A}_{D}^{\prime}: X_{o}^{\prime(n+1-k)} \rightarrow J^{\prime}$, the Abel map on the symmetric product of $X_{o}^{\prime}$. It suffices to show that $d \mathcal{A}_{D}^{\prime}$ is an injection at the base point $D$. The fibre of $\mathcal{A}_{D}^{\prime}$ over the identity is the projective space $\mathbb{P V}$ where

$$
V=\left\{f \in \mathbb{C}(X):(f) \geq-D \text { and } f\left(O_{j}\right)=f\left(O_{1}\right) \neq 0 \text { for every } j\right\} .
$$

Any $f \in V$ has degree $\leq k$, so unless it is constant it cannot take the same value at the $n+1$ distinct points $O_{j}$. Therefore $V=\mathbb{C}$ and it follows that $d \mathcal{A}_{D}^{\prime}$ is injective at $D$.

Next we must describe the image of $\gamma$ as a family of line bundles. Set $X_{A}^{\prime}=X^{\prime}-\lambda^{-1}(\{0, \infty\})$ and for each $j=1, \ldots k$ let $U_{j}$ be an open coordinate disc about $P_{j}$ for the coordinate $\zeta_{j}$. Further, let $V$ be a union of coordinate 
discs about the other points at $\lambda=0$ and assume $U_{1}, \ldots, U_{k}, V$ are mutually disjoint. Then

$$
\mathcal{U}=\left\{X_{A}^{\prime}, U_{1}, \ldots, U_{k}, V, \rho_{*} U_{1}, \ldots, \rho_{*} U_{k}, \rho_{*} V\right\}
$$

is a Leray cover for $X^{\prime}$ and any line bundle over it can be prescribed by a 1-cocycle for the open cover $\mathcal{U}$.

Lemma 5. Let $[c]: \mathbb{R}^{2 k} \rightarrow H^{1}\left(\mathcal{O}_{X^{\prime}}^{\times}\right)$be the map which gives the class of the 1-cocycle defined by

$$
c(z)= \begin{cases}\exp \left(z_{j} \zeta_{j}^{-1}\right) & \text { on } X_{A}^{\prime} \cap U_{j}, j=1, \ldots, k \\ 1 & \text { on } X_{A}^{\prime} \cap V\end{cases}
$$

and $\overline{\rho_{*} c(z)}=c(z)^{-1}$. Then $[c(z)]$ is the class of $\gamma(z)$.

Proof. Clearly $[c(z)]$ is the class of a degree zero line bundle in $J_{R}^{\prime}$. Consider, for each $m=1, \ldots, k$,

$$
\partial[c] /\left.\partial z_{m}\right|_{z=0}= \begin{cases}\zeta_{m}^{-1} & \text { on } X_{A}^{\prime} \cap U_{m} \\ 0 & \text { on every other 2-simplex from } \mathcal{U} .\end{cases}
$$

Recall (e.g. from [13]) that the Serre duality $H^{1}\left(\mathcal{O}_{X^{\prime}}\right) \simeq \Gamma\left(\Omega_{X^{\prime}}\right)^{*}$ identifies the class of this 1-cocycle with the residue map $f: \omega \mapsto \operatorname{res}_{P_{m}} \zeta_{m}^{-1} \omega$ for all $\omega \in \Gamma\left(\Omega_{X^{\prime}}\right)$. But

$$
\operatorname{res}_{P_{m}} \zeta_{m}^{-1} \omega=\left(\omega / d \zeta_{m}\right)\left(P_{m}\right)=\left.\left(\frac{\partial}{\partial \zeta_{m}} \int_{P_{m}}^{\zeta_{m}} \omega\right)\right|_{\zeta_{m}=0}
$$

It follows that $\left(\partial[c] / \partial z_{m}\right)(0)=\left(\partial \mathcal{A}_{P_{m}} / \partial \zeta_{m}\right)(0)$ whence $[c]$ and $\gamma$ are the same map.

Finally, before we prove theorem 1 let us make a few crucial observations concerning the canonical trivialisation of $G r_{k}\left(E^{\prime}\right)$ over $\mathcal{L}^{\prime} \otimes \gamma(z)$.

Any line bundle $\mathcal{L}^{\prime}$ over $X^{\prime}$ can be thought of as a line bundle $\mathcal{L}$ over $X$ equipped with fibre identifications $g_{j}: \mathcal{L}\left|O_{j} \rightarrow \mathcal{L}\right| O_{1}$, or equally, non-zero elements $s_{j} \in \mathcal{L} \mid O_{j}$, only fixed up to common scaling, for which $g_{j}$ identifies $s_{j}$ with $s_{1}$. From this point of view $\gamma(z)$ is the line bundle $L(z)=\pi(\gamma(z))$ with non-zero elements $s_{j}(z) \in L(z) \mid O_{j}$ which can be determined as follows. The 1-cocycle $c(z)$ equips $\gamma(z)$ with trivialising sections over each open set 
in $\mathcal{U}$. Let $s_{z}$ be the section over $X_{A}^{\prime}$ : it is only determined up to scaling. Then $s_{z}$ is a section of $L(z)$ over $X_{A}$ and $s_{j}(z)=s_{z} \mid O_{j}$. Notice that we may assume that $\overline{\rho_{*} s_{z}}=s_{z}^{-1}$. Now for any $\mathcal{L}^{\prime} \in N^{\prime}$, with non-zero global section $\sigma$ (recall this is unique up to scaling) the canonical trivialisation of $G r_{k}\left(E^{\prime}\right)$ over $\mathcal{L}^{\prime} \otimes \gamma(z)$ works as follows. Set $\mathcal{L}=\pi\left(\mathcal{L}^{\prime}\right)$ and $\mathcal{L}_{z}=\mathcal{L} \otimes L(z)$, then

$$
G r_{k}\left(\Gamma\left(\mathcal{L}_{z}\right)\right) \rightarrow G r_{k, n+1} ; \quad e_{1} \wedge \ldots \wedge e_{k} \mapsto v_{1} \wedge \ldots \wedge v_{k}
$$

where $v_{j} \in \mathbb{C}^{n+1}$ is given by

$$
v_{j}=\left(\left(e_{j} /\left(\sigma \otimes s_{z}\right)\right)\left(O_{1}\right), \ldots,\left(e_{j} /\left(\sigma \otimes s_{z}\right)\right)\left(O_{n+1}\right)\right)
$$

Proof of Theorem 1. The aim is to produce a map $F_{\lambda}: \mathbb{R}^{2 k} \rightarrow \Lambda G$ for which $F_{1}$ frames $\varphi$ and satisfies (3) for each $z_{m}, m=1, \ldots, k$. Set $\mathcal{L}_{z}=$ $\mathcal{L} \otimes L(z)$ for each $z \in \mathbb{R}^{2 k}$. Let $e_{1}^{z}, \ldots, e_{n+1}^{z}$ be a unitary frame for $\Gamma\left(\mathcal{L}_{z}\right)$ for which $e_{1}^{z}, \ldots, e_{k}^{z}$ span the $k$-plane $V=\Gamma\left(\mathcal{L}_{z}\left(-D_{\infty}\right)\right)$ and $e_{k+1}^{z}, \ldots, e_{n+1}^{z}$ span $V^{\perp}=\Gamma\left(\mathcal{L}_{z}\left(-\sum_{j=1}^{k} P_{j}\right)\right)$. A straightforward calculation with the trace norm (see [10] lemma 6) shows that $e, e^{\prime} \in \Gamma\left(\mathcal{L}_{z}\right)$ are orthogonal whenever $e^{\prime} \otimes \overline{\rho_{*} e}$ has divisor of zeroes at least $(\lambda)_{0}\left(\right.$ or $\left.(\lambda)_{\infty}\right)$, therefore these two spaces are orthogonal. Since any bundle over $\mathbb{R}^{2 k}$ is analytically trivial we can choose the $e_{j}^{z}$ to be analytic in $z$. Then $e_{1}^{z} \wedge \ldots \wedge e_{k}^{z}$ is the section of $G r_{k}\left(E^{\prime}\right)$ (pulled back to $\mathbb{R}^{2 k}$ ) which produces $\psi_{\mathcal{L}^{\prime}}$.

Now let $f_{1}^{z}, \ldots, f_{n+1}^{z}$ be the dual basis for $\Gamma\left(\mathcal{L}_{z}\right)^{*}$, and define the matrix $F_{\lambda}(z)$ to have entries

$$
F_{\lambda}(z)_{i j}=f_{i}^{0} e_{j}^{z} s_{z}^{-1} .
$$

To make sense of this, let $B$ denote the ring $\mathbb{C}\left[\lambda, \lambda^{-1}\right]$ and think of the $e_{j}^{z}$ as generators of the $B$-module $M_{z}=\Gamma\left(X_{A}, \mathcal{L}_{z}\right)$. This is free of rank $n+1$ since $\lambda_{*} \mathcal{L}_{z}$ is a trivial rank $n+1$ bundle over $\mathbb{P}^{1}$. We think of $f_{j}^{0}$ as generators for the dual module so that the entries of $F_{\lambda}(z)$ are elements of $B$. By the reality conditions $F_{\lambda}: \mathbb{R}^{2 k} \rightarrow U_{n+1}$ for $|\lambda|=1$. I claim that the projective frame $\left[F_{1}(z)\right]$ frames $\varphi(z)$, after possibly an isometry of $G r_{k, n+1}$. To see this notice that the only difference between (3.6) at $\lambda=1$ and (3.5) is that the former identifies $\oplus \mathcal{L} \mid O_{j}$ with $\mathbb{C}^{n+1}$ using the dual basis $\left\{f_{j}^{0}\right\}$ while the latter does this using the fibre elements $\left\{\sigma \mid O_{j}\right\}$. Either identification respects the Hermitian structure (up to scaling), hence the difference between them is a projective unitary transformation.

Now observe that

$$
\left(F_{\lambda}^{-1} \partial F_{\lambda} / \partial z_{m}\right)_{i j}=s_{z} f_{i}^{z} \partial\left(e_{j}^{z} s_{z}^{-1}\right) / \partial z_{m}
$$


If $e^{z} \in \Gamma\left(\mathcal{L}_{z}(-D)\right)$ is any smooth family of sections, for some divisor $D$, define

$$
\nabla_{z_{m}} e^{z}=s_{z} \partial\left(e^{z} s_{z}^{-1}\right) / \partial z_{m}
$$

Let $\pi_{V}^{\perp}$ be $B$-linear projection onto $V^{\perp} \otimes B$ with kernel $V \otimes B$. Then $F_{\lambda}$ will satisfy the conditions of lemma 3 for each $z=z_{m}, m=1, \ldots, k$, if

$$
\pi_{V}^{\perp}\left(\nabla_{z_{m}} e\right) \in \begin{cases}\lambda^{-1} V^{\perp} & \text { for } e \in V=\Gamma\left(\mathcal{L}_{z}\left(-D_{\infty}\right)\right) \\ \Gamma\left(\mathcal{L}_{z}\right) & \text { for } e \in V^{\perp}=\Gamma\left(\mathcal{L}_{z}\left(-\sum P_{j}\right)\right) .\end{cases}
$$

To see that this holds we recall from [10, lemma 7] that, whenever $e \in$ $\Gamma\left(\mathcal{L}_{z}(-D)\right)$ for some divisor $D$, then $\nabla_{z_{m}} e \in \Gamma\left(\mathcal{L}_{z}\left(P_{m}-D\right)\right)$. So for $e \in V$

$$
\nabla_{z_{m}} e \in \Gamma\left(\mathcal{L}_{z}\left(P_{m}-D_{\infty}\right)\right)=\mathbb{C}\left\langle\lambda^{-1} e_{0}, e_{1}, \ldots, e_{k}\right\rangle
$$

where $e_{0} \in V^{\perp}$ has divisor of zeros $(\lambda)_{0}-P_{m}$ (which is positive since $P_{m}$ is a ramification point of $\lambda$ ). Therefore $\pi_{V}^{\perp}\left(\nabla_{z_{m}} e\right)=\lambda^{-1} e_{0}$. On the other hand, for $e \in V^{\perp}$ we have

$$
\nabla_{z_{m}} e \in \Gamma\left(\mathcal{L}_{z}\left(-\sum_{j \neq m} P_{j}\right)\right) \subset \Gamma\left(\mathcal{L}_{z}\right)
$$

Therefore (3.9) holds, so by lemma $3 \varphi$ is harmonic is each variable $z_{m}$.

To see that $\varphi\left(z_{m}\right)$ is non-conformal we will show that the map

$$
A_{m}=\pi_{V} \nabla_{\bar{z}_{m}} \pi_{V}^{\frac{1}{V}} \nabla_{\bar{z}_{m}}: V \rightarrow V
$$

has nowhere vanishing trace. This shows that $\left(\partial \varphi / \partial \bar{z}_{m}, \partial \varphi / \partial \bar{z}_{m}\right)^{\mathbb{C}}$ vanishes nowhere, where this is the $(0,2)$-component of the complexification of the metric induced on $\mathbb{R}^{2}$ by $\varphi$. First observe that $\nabla_{\bar{z}_{m}} V \subset \Gamma\left(\mathcal{L}_{z}\left(Q_{m}-D_{\infty}\right)\right)$ so that we have

$$
V=\operatorname{ker}\left(\pi_{V}^{\frac{1}{V}} \nabla_{\bar{z}_{m}}\right) \oplus \mathbb{C}\left\langle\sigma_{0}^{z}\right\rangle
$$

where $\sigma_{0}^{z}$ generates $\Gamma\left(\mathcal{L}_{z}\left(-\hat{Q}_{m}\right)\right)$ and $\hat{Q}_{m}$ is the positive degree $n$ divisor $(\lambda)_{\infty}-Q_{m}$. Now observe that

$$
\nabla_{\bar{z}_{m}} \sigma_{0}^{z} \in V \oplus \mathbb{C}\left\langle\sigma_{1}\right\rangle
$$

where $\sigma_{1}$ generates $\Gamma\left(\mathcal{L}_{z}\left(-D_{m}-\sum P_{j}\right)\right) \subset V^{\perp}$ and $D_{m}$ is a degree $k-1$ positive divisor $D_{\infty}-Q_{m}$ since $Q_{m}$ is a ramification point of $\lambda$. Therefore we can write

$$
\pi_{V}^{\perp} \nabla_{\bar{z}_{m}} \sigma_{0}^{z}=a \sigma_{1}^{z}
$$


where $a$ depends only on $z$. Next, observe that

$$
\nabla_{\bar{z}_{m}} \sigma_{1}^{z} \in \Gamma\left(\mathcal{L}_{z}\left(Q_{m}-D_{m}-\sum P_{j}\right)\right)=V^{\perp} \oplus \mathbb{C}\left\langle\lambda \sigma_{0}^{z}\right\rangle .
$$

It can be shown, by a computation almost identical to the one in $[10, \mathrm{pp}$. 849-850], that

$$
\pi_{V} \nabla_{\bar{z}_{m}} \sigma_{1}^{z}=a^{-1} \lambda \sigma_{0}^{z}
$$

Finally, when we pass from $V \otimes B$ to $V$ (by evaluation at $\lambda=1$ ) we see, by choosing a basis for $V$ compatible with the splitting in (3.10), that the trace of $A_{m}: V \rightarrow V$ is 1 .

Remarks. 1. In fact similar methods can be used to show that

$$
\operatorname{Tr}\left(\pi_{V} \nabla_{\bar{z}_{l}} \pi_{V}^{\perp} \nabla_{\bar{z}_{m}}\right)=0 \text { for } l \neq m
$$

and therefore if we restrict $\varphi$ to the 2-plane with tangent $\sum a_{m} \partial / \partial z_{m}$ then $\varphi$ is non-conformal provided $\sum a_{m}^{2} \neq 0$.

2. What happens if $P_{m}$ has ramification index greater than 1? In that case the divisor $D_{m}-Q_{m}$ in (3.11) is positive, so that (3.11) becomes $\nabla_{\bar{z}_{m}} \sigma_{1}^{z} \subset V^{\perp}$. Hence $\operatorname{Tr}\left(\pi_{V} \nabla_{\bar{z}_{m}} \pi_{V}^{\perp} \nabla_{\bar{z}_{m}}\right)=0$ i.e. $\varphi\left(z_{m}\right)$ is a minimal surface (i.e. conformal harmonic). If every $P_{1}, \ldots, P_{k}$ has this property then $\varphi$ is minimal when restricted to any 2 -plane. Indeed, we can raise the isotropy order (in the sense of [17]) of $\varphi\left(z_{m}\right)$ further by increasing the ramification index of $P_{m}$, in the same way we see this in [10, Thm. 4, p. 847].

\subsection{Maps into $\mathbb{P} U_{n+1}$.}

In this case for the spectral data $(X, \lambda)$ we do not assume that $\lambda$ has any double zeroes. Indeed, for simplicity we will assume that $\lambda$ only has simple zeroes $P_{1}, \ldots, P_{n+1}$ with corresponding poles $\left.Q_{j}=\rho_{(} P_{j}\right)$. But the singularisation $X^{\prime}$ we will use is not the same as in the previous section. We want $X^{\prime}$ to have two singular points $O$ and $S$, corresponding to two divisors: $\mathfrak{o}=O_{1}+\ldots+O_{n+1}$, the divisor of zeroes of $\lambda-1$, and $\mathfrak{s}=S_{1}+\ldots+S_{n+1}$, the divisor of zeroes of $\lambda+1$. Each divisor provides us with a singularisation of X: call them $X_{0}$ and $X_{5}$ respectively. Then $X^{\prime}$ is a further singularisation of each of these. It follows that we have a commuting diagram of generalised 
Jacobi varieties:

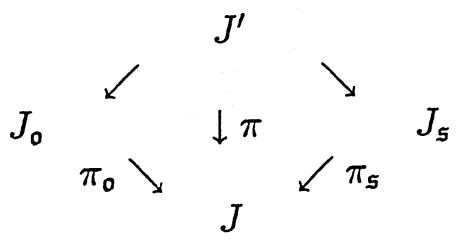

Both $\pi_{0}^{*} E$ and $\pi_{s}^{*} E$ are projectively trivial. Let us denote by $\tau_{O}$ and $\tau_{S}$ the two sections of $\mathbb{P} F r\left(\pi^{*} E\right)$ obtained by pulling back, respectively, the canonical sections of $\mathbb{P} F r\left(\pi_{0}^{*} E\right)$ and $\mathbb{P} F r\left(\pi_{s}^{*} E\right)$. If we now restrict our attention to $E^{\prime}=\pi^{*} E \mid N^{\prime}$ then $\tau_{O}$ and $\tau_{S}$ are sections of $\mathbb{P} U\left(E^{\prime}\right)$. It follows that we have a change of frame map

$$
\Psi: N^{\prime} \rightarrow \mathbb{P} U_{n+1}, \quad \tau_{O}=\Psi \tau_{S}
$$

For each $\mathcal{L}^{\prime} \in N^{\prime}$ we define $\Psi_{\mathcal{L}^{\prime}}$ by precomposing $\Psi$ with the isomorphism $J_{R}^{\prime} \simeq N^{\prime}$ which identifies the identity in $J_{R}^{\prime}$ with $\mathcal{L}^{\prime}$.

Since each zero $P_{j}$ of $\lambda$ is assumed to be simple we can use $\lambda$ as a local coordinate (which we name $\zeta_{j}$ ) about each $P_{j}$. Define $\gamma: \mathbb{R}^{2 n} \rightarrow J_{R}^{\prime}$ to be the unique homomorphism satisfying (3.3) for $j=1, \ldots, n$. Then $\gamma$ is an immersion by lemma 4 (adapted to the case where $X^{\prime}$ has two singular points).

Theorem 2. For each $\mathcal{L}^{\prime} \in N^{\prime}$, the map $\varphi=\Psi_{\mathcal{L}^{\prime}} \circ \gamma: \mathbb{R}^{2 n} \rightarrow \mathbb{P} U_{n+1}$ is pluri-harmonic. Moreover, $\varphi\left(z_{m}\right)$ is non-conformal for $m=1, \ldots, n$.

Proof. The proof is much the same as the proof of theorem 1. Let $L: \mathbb{R}^{2 n} \rightarrow$ $J$ be defined by $L=\pi \circ \gamma$, set $\mathcal{L}=\pi\left(\mathcal{L}^{\prime}\right)$ and define $\mathcal{L}_{z}=\mathcal{L} \otimes L(z)$. Let $\left\{e_{i}^{z}\right\}$ be a unitary basis for $\Gamma\left(\mathcal{L}_{z}\right)$ and take $\left\{f_{j}^{z}\right\} \subset \Gamma\left(\mathcal{L}_{z}\right)^{*}$ to be the dual basis. As earlier, $L(z)$ admits a trivialising section $s_{z}$ over $X-\lambda^{-1}(\{0, \infty\})$ with the properties: (i) $s_{z} \exp \left(z_{j} \lambda^{-1}\right)$ is non-vanishing and holomorphic about $P_{j}$ while $s_{z} \exp \left(-\bar{z}_{j} \lambda\right)$ is holomorphic and non-vanishing about $Q_{j}$, (ii) $\overline{\rho_{*} s_{z}}=s_{z}^{-1}$. As before we introduce the matrix $F_{\lambda}(z)$ with entries

$$
F_{\lambda}(z)_{i j}=\left(f_{i}^{0} e_{j}^{z} s_{z}^{-1}\right) .
$$

I claim that: (a) after possibly an isometry, $\varphi$ is framed in $\mathbb{P} U_{n+1}$ by $\left[F_{-1} F_{1}^{-1}\right]$, where the square brackets denote the class in $\mathbb{P} U_{n+1}$; (b) $\left[F_{\lambda} F_{1}^{-1}\right]$ is an extended frame for a pluri-harmonic map of $\mathbb{R}^{2 n}$ into $\mathbb{P} U_{n+1}$. 
For (a), let $\left[F_{\lambda}\right]$ denote the map into $\mathbb{P} U_{n+1}$ at each $|\lambda|=1$ and let $\left[e_{z}\right]$ denote the projective frame determined by the $e_{j}^{z}$. By the same reasoning as in the proof of the previous theorem, there exist $a, b \in \mathbb{P} U_{n+1}$ for which

$$
\left[e_{z}\right]=\tau_{O}(\gamma(z)) a\left[F_{1}(z)\right]=\tau_{S}(\gamma(z)) b\left[F_{-1}(z)\right]
$$

for all $z$, so that $\Psi \circ \gamma=b\left[F_{-1} F_{1}\right] a^{-1}$. Since the metric on $\mathbb{P} U_{n+1}$ is biinvariant $\left[F_{-1} F_{1}^{-1}\right]$ differs from $\varphi$ by an isometry.

For (b), we compute, for each $m=1, \ldots, n$,

$$
F_{\lambda}^{-1} \partial F_{\lambda} / \partial z_{m}=\left(a_{i j}^{(m)}\right) ; \quad a_{i j}^{(m)}=f_{i}^{z} \nabla_{z_{m}} e_{j}^{z}
$$

(cf. (3.7) and (3.8)). A computation similar to the one earlier shows that

$$
\nabla_{z_{m}} e \in \begin{cases}\Gamma\left(\mathcal{L}_{z}\right) & \text { for } e \in \Gamma\left(\mathcal{L}_{z}\left(-P_{m}\right)\right) ; \\ \lambda^{-1} \Gamma\left(\mathcal{L}_{z}\right) \oplus \Gamma\left(\mathcal{L}_{z}\right) & \text { otherwise. }\end{cases}
$$

It follows that $F_{\lambda}^{-1} \partial F_{\lambda} / \partial z_{m}$ has linear dependence on $\lambda^{-1}$. So if we define $\tilde{F}_{\lambda}=F_{\lambda} F_{1}^{-1}$ then

$$
\tilde{F}_{\lambda}^{-1} \frac{\partial \tilde{F}_{\lambda}}{\partial z_{m}}=\left(1-\lambda^{-1}\right) \tilde{A}_{m}
$$

for some matrix function $\tilde{A}_{m}$ independent of $\lambda$, whence the map is harmonic.

Now we will check that $\varphi\left(z_{m}\right)$ is non-conformal. Notice that means checking that

$$
\left(\partial \varphi / \partial z_{m}, \partial \varphi / \partial z_{m}\right)^{\mathbb{C}}=\operatorname{Tr}\left(\tilde{A}_{m}^{2}\right)
$$

is not identically zero. First, let us note that if we write

$$
F_{\lambda}^{-1} \partial F_{\lambda} / \partial z_{m}=\lambda^{-1} A_{m}+B_{m}
$$

then a simple calculation shows that $\tilde{A}_{m}=\operatorname{AdF}_{1}\left(-2 \mathrm{~A}_{\mathrm{m}}\right)$ so that $\operatorname{Tr}\left(\tilde{A}_{m}^{2}\right)=$ $\operatorname{Tr}\left(4 A_{m}^{2}\right)$. Now fix the basis $\left\{e_{j}^{z}\right\}$ so that $e_{j}^{z} \in V=\Gamma\left(\mathcal{L}_{z}\left(-P_{m}\right)\right)$ for $j \neq m$ and $e_{m}^{j}$ generates $V^{\perp}=\Gamma\left(\mathcal{L}_{z}\left(-\hat{Q}_{m}\right)\right)$, where $\hat{Q}_{m}$ is the positive divisor $(\lambda)_{\infty}-Q_{m}$. Notice from (3.12) that $\nabla_{z_{m}} V \subset \Gamma\left(\mathcal{L}_{z}\right)$. This means that, in this basis, all the columns of $A_{m}$ except possibly the $m$-th are zero. Therefore, $\operatorname{Tr}\left(A_{m}^{2}\right)=A_{m, m m}^{2}$, where $A_{m, m m}$ is the $m$-th entry of the $m$-th column. To compute this, we first notice that

$$
\nabla_{z_{m}} e_{m}^{z} \in \Gamma\left(\mathcal{L}_{z}\left(P_{m}-\hat{Q}_{m}\right)\right)=\mathbb{C}\left\langle\lambda^{-1} e^{z}, e_{m}^{z}\right\rangle
$$


where $e^{z}$ generates $\Gamma\left(\mathcal{L}_{z}\left(-\hat{P}_{m}\right)\right)$ with $\hat{P}_{m}=(\lambda)_{0}-P_{m}$. But

$$
\Gamma\left(\mathcal{L}_{z}\left(-\hat{P}_{m}\right)\right) \not \subset V
$$

so that $f_{m}^{z}\left(e^{z}\right) \neq 0$, therefore $A_{m, m m} \neq 0$ whence $\varphi\left(z_{m}\right)$ is non-conformal.

Remark. One knows from [3] that all non-conformal tori in $\mathbb{P} U_{2}$ are of semisimple finite type and I claim the construction above will give them all. But in general it is not clear to what extent the maps of semisimple finite type account for the harmonic tori. As with the case of maps into $G r_{k, n+1}$, we can increase the isotropy order of $\varphi\left(z_{m}\right)$ by increasing the ramification index of $P_{m}$. In particular, $\varphi\left(z_{m}\right)$ is minimal whenever $P_{m}$ is a ramification point. For in that case $\hat{P}_{m}$ has support at $P_{m}$ so (3.13) becomes $\Gamma\left(\mathcal{L}_{z}\left(-\hat{P}_{m}\right)\right) \subset V=\operatorname{ker} f_{m}^{z}$, whence $A_{m, m m}=0$.

\subsection{Equivariant maps.}

For any subgroup $S \subset \mathbb{R}^{2 k}$ we will say a map $\varphi: \mathbb{R}^{2 k} \rightarrow G r_{k, n+1}$ is $S$ equivariant if there exists a homomorphism $h: S \rightarrow \mathbb{P} U_{n+1}$ for which $\varphi(z+$ $s)=h(s) \varphi(z)$ for all $z \in \mathbb{R}^{2 k}$ and $s \in S$.

Proposition 2. Let $\varphi=\psi \circ \gamma: \mathbb{R}^{2 k} \rightarrow J_{R}^{\prime} \rightarrow G r_{k, n+1}$ be given by theorem 1 and let $S \subset \mathbb{R}^{2 k}$ be the subgroup covering $\gamma\left(\mathbb{R}^{2 k}\right) \cap H_{0}$. Then $\varphi$ is $S$ equivariant.

Proof. This follows at once from lemma 1. From the proof of theorem 1 we know that $\psi$ is derived by canonical trivialisation of a section of $G r_{k}(E)$ lifted to $G r_{k}\left(E^{\prime}\right)$. By lemma 1 and the definition of $S, \varphi(z+s)=\gamma(s) \psi(\gamma(z))$ for $z \in \mathbb{R}^{2 k}$ and $s \in S$, where we have assumed the identification of $H_{0}$ with $H \subset \mathbb{P} U_{n+1}$.

Clearly the same result holds for the maps $\varphi: \mathbb{R}^{2 n} \rightarrow \mathbb{P} U_{n+1}$ constructed by theorem 2 , where now we have a homomorphism $S \rightarrow \operatorname{Isom}\left(\mathbb{P} U_{n+1}\right) \simeq$ $\mathbb{P} U_{n+1} \times \mathbb{P} U_{n+1}$. We will say the map $\varphi$ is totally equivariant if it has maximal equivariance group ( $\mathbb{R}^{2 k}$ or $\mathbb{R}^{2 n}$ as appropriate).

Corollary 1. If the spectral curve $X$ for $\varphi$ has genus $g<2 k$ (resp. $g<2 n$ ) then $\varphi$ is an $\mathbb{R}^{2 k-g}$-equivariant map into $G r_{k, n+1}$ (resp. an $\mathbb{R}^{2 n-g}$. equivariant map into $\left.\mathbb{P} U_{n+1}\right)$. In particular, when $X$ is the Riemann sphere the map is totally equivariant. 
Proof. The kernel of $d(\pi \circ \gamma)$ has dimension $2 k-g$ (resp. $2 n-g)$.

\section{The $\theta$-line bundle and algebraic maps.}

The aim of this section is to show that the maps produced from the canonical trivialisation can be written down using sections of the pullback of the $\theta$-line bundle to $J^{\prime}$. We then look at the possibility that the $\varphi$ is algebraic (which happens precisely when $\gamma$ is algebraic). It turns out, at least for maps into $\mathbb{C P}^{n}$, that this forces $\varphi$ to be doubly periodic and therefore give harmonic 2 -tori.

\subsection{The pullback of the $\theta$-line bundle to $J^{\prime}$.}

Let $\mathcal{T}$ denote the $\theta$-line bundle over $J$. It has, up to scaling, a unique global section $\theta$ which we will also think of as the classical Riemann $\theta$-function over $\mathbb{C}^{g}$ i.e. we will assume we have made all the appropriate identifications so that $J \simeq \mathbb{C}^{g} / \Lambda$ where $\Lambda \simeq H_{1}(X, \mathbb{Z})$. We want to describe $\Gamma\left(\pi^{*} \mathcal{T}\right)$ explicitly. General theory (see e.g. [6, p. 128]) tells us that the space $\Gamma\left(\pi^{*} \mathcal{T}\right)$ of globally algebraic sections should be isomorphic to the coordinate ring of any fibre of $\pi: J^{\prime} \rightarrow J$ as a vector space. We will actually compute a spanning set of sections for $\Gamma\left(\pi^{*} \mathcal{T}\right)$.

First, notice that we can describe $J^{\prime}$ as a fibre product over $J$ :

$$
J^{\prime} \simeq J_{1} \times{ }_{J} \ldots \times_{J} J_{n}
$$

where $J_{k}$ is the generalised Jacobian for the modulus $O_{1}+O_{k+1}$. Each $J_{k}$ is a $\mathbb{C}^{\times}$-bundle over $J$ once we have fixed an isomorphism of $H_{k} \simeq J_{k} / J$ with $\mathbb{C}^{\times}$. We fix this so that for any $L \in J$ the group $\mathbb{C}^{\times}$acts on $\operatorname{Hom}\left(L\left|O_{k+1}, L\right| O_{1}\right)$ by multiplication. Every $\mathbb{C}^{\times}$-bundle is characterised by its pullback to $X$ via the Abel map $\mathcal{A}: X \rightarrow J$ and with this choice $J_{k}$ is characterised by $\mathcal{A}^{*} J_{k}=\mathcal{A}\left(O_{1}-O_{k+1}\right)$ (cf. [13, VII no. 9]). For each $k$ let $\pi_{k}: J^{\prime} \rightarrow J_{k}$ denote the natural fibration. To describe $\Gamma\left(\pi^{*} \mathcal{T}\right)$ we need to introduce $t_{k}: J \rightarrow J$, the translation by $\mathcal{A}\left(O_{1}-O_{k+1}\right)$, and $s_{k}$, the tautological section of $\pi^{*} J_{k} \rightarrow J^{\prime}$. We will denote by $t_{k}^{m}$ the $m$-th iterate of the translation $t_{k}$ (i.e. translation by $\mathcal{A}\left(m O_{1}-m O_{k+1}\right)$ ).

Proposition 3. There is a vector space isomorphism between $\mathbb{C}\left[x_{1}, x_{1}^{-1}\right] \otimes$ $\ldots \otimes \mathbb{C}\left[x_{n}, x_{n}^{-1}\right]$ and $\Gamma\left(\pi^{*} \mathcal{T}\right)$ given by

$$
x_{1}^{k_{1}} \ldots x_{n}^{k_{n}} \mapsto \theta_{k_{1}, \ldots, k_{n}}=s_{1}^{k_{1}} \otimes \ldots \otimes s_{n}^{k_{n}} \otimes \pi^{*}\left(t_{1}^{-k_{1}} \ldots t_{n}^{-k_{n}}\right)^{*} \theta .
$$


Proof. First we must establish that every $\theta_{k_{1}, \ldots, k_{n}}$ belongs to $\Gamma\left(\pi^{*} \mathcal{T}\right)$. To simplify notation, define $\xi=\sum_{j=1}^{n} \mathcal{A}\left(k_{j} O_{j+1}-k_{j} O_{1}\right)$, then $t_{\xi}=t_{1}^{-k_{1}} \ldots t_{n}^{-k_{n}}$. Let us show that

$$
t_{\xi}^{*} \mathcal{T} \simeq \mathcal{T} \otimes J_{1}^{k_{1}} \otimes \ldots \otimes J_{n}^{k_{n}}
$$

where, with a slight abuse of notation, we are thinking of each $J_{m} \rightarrow J$ as the associated line bundle to the $\mathbb{C}^{\times}$-bundle. Since the pullback by the Abel map induces an isomorphism $H^{1}\left(X, \mathcal{O}_{X}^{\times}\right) \simeq H^{1}\left(J, \mathcal{O}_{J}^{\times}\right)$it suffices to show that $\mathcal{A}^{*}\left(t_{\xi}^{*} \mathcal{T}\right)=\mathcal{A}^{*} \mathcal{T} \otimes \xi$, where we use $\xi$ here to denote the line bundle over $X$ for the point $\xi \in J$. But this follows immediately from Riemann's vanishing theorem.

Now if we pull back (4.2) to $J^{\prime}$ and use the fact that $s_{k}$ trivialises $\pi^{*} J_{k}$ we see that $\pi^{*} t_{\xi}^{*} \mathcal{T} \simeq \mathcal{T}$ and tensoring by $s_{1}^{k_{1}} \otimes \ldots \otimes s_{n}^{k_{n}}$ identifies $\pi^{*} t_{\xi}^{*} \theta$ with $\theta_{k_{1}, \ldots, k_{n}}$.

To prove that these span $\Gamma\left(\pi^{*} \mathcal{T}\right)$ we take the point of view that each section is a holomorphic function on the universal cover $\mathbb{C}^{g+n}$ of $J^{\prime}$ satisfying certain functional equations. To identify $J^{\prime}$ with $\mathbb{C}^{g+n} / \Lambda^{\prime}$, where $\Lambda^{\prime} \simeq$ $H^{1}\left(X^{\prime}-\{O\}, \mathbb{Z}\right)$, we augment the standard first homology basis $\left\{a_{j}, b_{j}\right.$ : $j=1, \ldots, g\}$ of $X$ with a positively oriented cycle $a_{g+j}$ enclosing a small disc about each $O_{j+1}, j=1, \ldots, n$. Then $\left\{\oint_{a_{j}}: j=1, \ldots, g+n\right\}$ is a basis for $\Gamma\left(\Omega_{X^{\prime}}\right)^{*}$. A function $f: \mathbb{C}^{g+n} \rightarrow \mathbb{C}$ corresponds to a section of $\pi^{*} \mathcal{T}$ if and only if

$$
\left(f / \pi^{*} \theta\right)(\tilde{Z}+\lambda)=\left(f / \pi^{*} \theta\right)(\tilde{Z}), \text { for all } \lambda \in \Lambda^{\prime}, \tilde{Z} \in \mathbb{C}^{g+n} .
$$

It is easy to compute that

$$
\begin{aligned}
& \theta_{k_{1}, \ldots, k_{n}}\left(z_{1}, \ldots, z_{g+n}\right) \\
& =\exp \left(2 \pi i \sum_{j=1}^{n} k_{j} z_{g+j}\right) \theta\left(Z+\sum_{j=1}^{n} k_{j} \mathcal{A}\left(O_{j+1}-O_{1}\right)\right)
\end{aligned}
$$

where $Z=\left(z_{1}, \ldots, z_{g}\right)$ and the projection $\mathbb{C}^{g+n} \rightarrow \mathbb{C}^{g}$ covering $J^{\prime} \rightarrow J$ has fibre coordinates $z_{g+1}, \ldots, z_{g+n}$. A straightforward but tedious Fourier decomposition argument shows that all holomorphic functions $f(\tilde{Z})$ satisfying (4.3) must be a linear combination of the functions above (cf. the proof that the classical $\theta$-function spans $\Gamma(\mathcal{T})$ in e.g. [5]).

We want to use this to produce a more explicit expression for the maps $\psi_{\mathcal{L}^{\prime}}: N^{\prime} \rightarrow G r_{k, n+1}$ which appear in theorem 1. To this end, fix $\mathcal{L}^{\prime} \in N^{\prime}$ 
and set $\mathcal{L}=\pi(\mathcal{L})$. Let us begin by considering, for $L^{\prime} \in J_{R}^{\prime}$ and $L=\pi\left(L^{\prime}\right)$, the maps

$$
\begin{aligned}
& \mathbb{P} \Gamma(\mathcal{L} \otimes L) \rightarrow \mathbb{C P}^{n}, \\
& e_{L} \mapsto\left[\left(e_{L} /\left(\sigma \otimes s_{L}\right)\right)\left(O_{1}\right), \ldots,\left(e_{L} /\left(\sigma \otimes s_{L}\right)\right)\left(O_{n+1}\right)\right]
\end{aligned}
$$

derived from the canonical trivialisation (cf. equation (3.5)). Recall that in this expression $\sigma$ generates $\Gamma\left(\mathcal{L}^{\prime}\right)$ and $s_{L}$ is a trivialising section for $\mathcal{L}^{\prime} \otimes L^{\prime}$ over $X-\lambda^{-1}(\{0, \infty\})$. We are only interested in the case where $e_{L}$ generates $\Gamma(\mathcal{L}(-\tilde{D}) \otimes L)$ for some positive divisor $\tilde{D}$ of degree $n$. In that case there is a positive divisor $D$ of degree $g$ such that $\mathcal{L}(-\tilde{D}) \simeq \mathcal{O}_{X}(D)$. Let $\mathcal{T}_{D}$ be the translate of $\mathcal{T}$ for which $\mathcal{A}^{*} \mathcal{T}_{D} \simeq \mathcal{O}_{X}(D)$, then the previous proposition holds equally well for $\mathcal{T}_{D}$ with $\theta(Z)$ replaced by the appropriate translate $\theta\left(Z+\kappa_{D}\right)$. By (4.1) the monomials $1, x_{1}, \ldots, x_{n}$ have images in $\Gamma\left(\pi^{*} \mathcal{T}_{D}\right)$ which we will simply call $\theta_{0}\left(\tilde{Z}+\kappa_{D}\right), \theta_{1}\left(\tilde{Z}+\kappa_{D}\right), \ldots, \theta_{n}\left(\tilde{Z}+\kappa_{D}\right)$ (here we lift $\kappa_{D} \in \mathbb{C}^{g}$ up to $\mathbb{C}^{g+n}$ using the first $g$ coordinates of the latter).

Proposition 4. For $e_{L} \in \Gamma(\mathcal{L}(-\tilde{D}) \otimes L)$ as described above the map (4:4) can also be written

$$
e_{L} \mapsto\left[c_{0} \theta_{0}\left(\tilde{Z}+\kappa_{D}\right), \ldots, c_{n} \theta_{n}\left(\tilde{Z}+\kappa_{D}\right)\right]
$$

where $\mathbb{C}^{g+n} \rightarrow J^{\prime} \rightarrow J$ maps $\tilde{Z} \mapsto L$ and $c_{0}, c_{1}, \ldots, c_{n}$ are constants which depend only on $\mathcal{L}^{\prime}$ and $\tilde{D}$.

Proof. Fix the base point for $\mathcal{A}: X \rightarrow J$ to be $O_{1}$. Then $e_{L}$ can be identified with $\mathcal{A}^{*} \theta\left(Z+\kappa_{D}\right)$ and so $e_{L} \mid O_{k}$ is identified with

$$
\theta\left(\mathcal{A}\left(O_{k}\right)+Z+\kappa_{D}\right)=t_{k}^{-1 *} \theta\left(Z+\kappa_{D}\right),
$$

since $\mathcal{A}\left(O_{k}\right)=\mathcal{A}\left(O_{k}-O_{1}\right)$ with this choice of base point. Now we observe that we have the tautological sections

$$
s_{k}: J^{\prime} \rightarrow \pi^{*} J_{k}, \quad L^{\prime} \rightarrow\left(L^{\prime}, s_{L}\left|O_{1} \otimes s_{L}^{-1}\right| O_{k}\right) .
$$

It follows that

$$
\left[\left(e_{L} \otimes s_{L}^{-1}\right)\left|O_{1}, \ldots,\left(e_{L} \otimes s_{L}^{-1}\right)\right| O_{n+1}\right]=\left[\theta_{0}\left(\tilde{Z}+\kappa_{D}\right), \ldots, \theta_{n}\left(\tilde{Z}+\kappa_{D}\right)\right] .
$$

We obtain (4.5) by observing that the additional information $\sigma \mid O_{1}, \ldots$, $\sigma \mid O_{n+1}$ is independent of $L^{\prime}$ and only contributes constants $c_{0}, \ldots, c_{n}$. 
Let us apply this to write down the formula for the map $\varphi: \mathbb{R}^{2 k} \rightarrow$ $G r_{k, n+1}$ with spectral data $(X, \lambda, \mathcal{L})$. For simplicity set $z=\left(z_{1}, \ldots, z_{k}\right)$ and, for $j=1, \ldots, k$ let $U_{j} \in \mathbb{C}^{g+n}$ be the tangent vector $d \gamma\left(\partial / \partial z_{j}\right)$ at $z=0$. Let us simplify notation by setting $U \cdot z=\sum_{j=1}^{k} U_{j} z_{j}$ : this maps $\mathbb{C}^{k}$ into $\mathbb{C}^{g+n}$. The space $\Gamma\left(\mathcal{L}\left(-D_{\infty}\right)\right)$ is spanned by non-zero sections $v_{1}, \ldots, v_{k}$ for which $v_{j}$ has divisor of zeroes $\hat{Q}_{j}=(\lambda)_{\infty}-Q_{j}$ : these will not be orthogonal. In the linear equivalence class for $\left.\mathcal{L}\left(-\hat{Q}_{j}\right)\right)$ there is a unique positive divisor $D_{j}$ : let $\kappa_{j} \in \mathbb{C}^{g}$ be such that $\theta\left(\mathcal{A}_{P_{0}}(P)+\kappa_{j}\right)$ has divisor of zeroes $D_{j}$. Then

$$
\varphi(z)=v_{1}(z) \wedge \ldots \wedge v_{k}(z)
$$

where

$$
v_{j}(z)=\left(c_{0 j} \theta_{0}\left(U \cdot z+\bar{U} \cdot \bar{z}+\kappa_{j}\right), \ldots, c_{n j} \theta_{n}\left(U \cdot z+\bar{U} \cdot \bar{z}+\kappa_{j}\right)\right) .
$$

The constants $c_{i j}$ can be computed using $\theta$-functions but the formula is not elementary.

\subsection{Algebraic harmonic tori.}

From the results above (see also [11]) it is clear that we can obtain harmonic tori whenever the map $\gamma: \mathbb{R}^{2 k} \rightarrow J_{R}^{\prime}$ has two independent periods. In general this is a difficult condition to study, since the image of $\gamma$ need not be algebraic and in general we must solve some transcendental equations in the moduli of $(X, \lambda)$. Here we examine what happens when the image of $\gamma$ is algebraic. For simplicity we will work with the case of maps into $\mathbb{C P}^{n}$. We will say a map $\varphi: \mathbb{R}^{2} \rightarrow \mathbb{C P}^{n}$ is algebraic if the image of the complexification of $\gamma$ is an algebraic subgroup of $J^{\prime}$. We can describe this complex group homomorphism $\gamma^{\mathbb{C}}$, as follows. Recall that to construct maps into $\mathbb{C P}^{n}$ with spectral data $(X, \lambda)$ we first single out a zero $P_{1}$ of $\lambda$ (of at least degree two) and the corresponding pole $Q_{1}=\rho_{*} P_{1}$. Let $\mathcal{A}_{P_{1}}^{\prime}, \mathcal{A}_{Q_{1}}^{\prime}: X^{\prime} \rightarrow J^{\prime}$ be the (rational) Abel maps for these base points. Then $\gamma^{\mathbb{C}}: \mathbb{C}^{2} \rightarrow J^{\prime}$ is the unique homomorphism of complex groups with the property that

$$
\left(\partial \gamma^{\mathbb{C}} / \partial z\right)(0,0)=\left(\partial \mathcal{A}_{P_{1}}^{\prime} / \partial \zeta\right)\left(P_{1}\right) ; \quad\left(\partial \gamma^{\mathbb{C}} / \partial w\right)(0,0)=\left(\partial \mathcal{A}_{Q_{1}}^{\prime} / \partial \eta\right)\left(Q_{1}\right),
$$

for coordinates $(z, w)$ on $\mathbb{C}^{2}$ and local parameters $\zeta=\sqrt{\lambda}$ about $P_{1}$ and $\eta=\sqrt{\lambda^{-1}}$ about $Q_{1}$. We may choose these square roots so that $\overline{\rho_{*} \zeta}=-\eta^{-1}$. It follows that $\gamma^{\mathbb{C}}(z, \bar{z})$ is the real homomorphism $\gamma(z)$. We will denote the image of $\gamma^{\mathbb{C}}$ by $M \subset J^{\prime}$ and let $M_{R}$ be the image of $\gamma$ (which is the connected component of the identity of $M \cap J_{R}^{\prime}$ ). One knows from [11, p. 520] that 
$M$ is two dimensional unless $X$ is the Riemann sphere and $\lambda$ has degree two (for that is the only case for which $\operatorname{dim} J^{\prime}<2$ ).

Proposition 5. If $M \subset J^{\prime}$ is an algebraic subgroup then it must be one of three types:

(A) $M \simeq \mathbb{C}^{\times} \times \mathbb{C}^{\times}$. In this case $X$ is the Riemann sphere and the harmonic $\operatorname{map} \varphi: M_{R} \rightarrow \mathbb{P}^{n}$ is $S^{1} \times S^{1}$-equivariant.

(B) $M$ is a $\mathbb{C}^{\times}$-extension of an elliptic curve. In this case $X$ is hyperelliptic and the map $\varphi$ is $S^{1}$-equivariant.

(C) $M$ is compact i.e. an abelian surface.

In each case $M_{R}$ is a real two torus.

Proof. Set $\hat{S}=M \cap H_{\mathrm{o}}$ and let $S$ be its connected component of the identity. Since $M$ is algebraic $S$ is a product of $\mathbb{C}^{\times}$'s and $\hat{S} / S$ is a finite group. We know immediately from proposition 2 that the map $\varphi: M_{R} \rightarrow \mathbb{P}^{n}$ must be equivariant for the real group $\hat{S} \cap M_{R}$. Now set $A=M / S$, then we have exact sequences

$$
1 \rightarrow S \rightarrow M \rightarrow A \rightarrow 1, \quad 1 \rightarrow \hat{S} / S \rightarrow A \rightarrow \pi(M) \rightarrow 1 .
$$

Notice that $\pi(M) \subset J$ must be an abelian variety, possibly trivial, and therefore so is $A$. Now we have only three possibilities for the dimension of $S$ :

(A) $\operatorname{dim} S=2$. It follows that $M=S$ (since $M$ is connected) whence $M \simeq \mathbb{C}^{\times} \times \mathbb{C}^{\times}$, so $\varphi$ is $S^{1} \times S^{1}$ equivariant. But this means $\pi(M)=1$. Now we observe that $M$ is tangent to $\mathcal{A}_{P_{1}+Q_{1}}^{\prime}\left(X^{\prime(2)}\right) \subset J^{\prime}$ (where $X^{\prime(2)}$ denotes the symmetric product) and one knows that $d \pi \circ d \mathcal{A}^{\prime}=d \mathcal{A}$. It follows that $\pi(M)$ is tangent to $\mathcal{A}_{P_{1}+Q_{1}}\left(X^{(2)}\right) \subset J$. Therefore to have $\pi(M)=1$ it must be that $J$ is the trivial group i.e. $X$ is the Riemann sphere.

(B) $\operatorname{dim} S=1$. It follows that $\operatorname{dim} A=1$ i.e. it is an elliptic curve so $M$ is a $\mathbb{C}^{\times}$-extension and $\varphi$ is $S^{1}$ equivariant. We also have $\operatorname{dim} \pi(M)=1$. But one easily sees, by identifying $\Gamma\left(\Omega_{X}\right)^{*}$ with the tangent space to $J$ at the identity, that the image of $d \mathcal{A}_{P_{1}+Q_{1}}$ is the annihilator of $\Gamma\left(\Omega_{X}\left(-P_{1}-Q_{1}\right)\right.$, so this must have codimension one in $\Gamma\left(\Omega_{X}\right)$. It follows that $P_{1}+Q_{1}$ is the divisor of zeroes of a rational function on $X$ (see e.g. [6, p. 341]) i.e. $X$ is hyperelliptic. 
(C) $\operatorname{dim} S=0$. In this case $M$ equals $A$ and is therefore isogenous to $\pi(M)$, hence $M$ is an abelian surface.

In each case $S \cap J_{R}^{\prime}$ and $A \cap J_{R}^{\prime}$ are real tori, therefore $M_{R}$ is a real 2 -torus.

Remarks. The algebraic tori are a very special class, for in this case the $\theta$-functions which occur in (4.5) will reduce to $\theta$-functions for the generalised abelian variety $M$. In case (A) $M$ is the generalised Jacobian of a rational curve with two nodes, while in case (B) $M$ is the generalised Jacobian of an elliptic curve with one node (but note that in case (C) $M$ need not be the Jacobi variety of any curve). However, the case of totally equivariant minimal tori in $\mathbb{C P}^{n}$ (i.e. case (A) for conformal maps) has already been treated by Jensen-Liao [8] using a parameterisation due to Kenmotsu [9]. I don't believe that the spectral curve approach adds much to this. One knows from [10] that a harmonic map $\varphi: \mathbb{R}^{2} \rightarrow \mathbb{C P}^{n}$ is totally equivariant if and only if its spectral curve is the Riemann sphere and since $J^{\prime}$ is a product of $\mathbb{C}^{\times}$'s it follows that $\varphi$ is algebraic if and only if it is a two torus. One can write down fairly explicitly the conditions which guarantee that $M$ is algebraic. They amount to a system of linear equations, parameterised by the moduli of the pair $(X, \lambda)$, which must have rational solutions (the reader interested in seeing a description of the moduli space $(X, \lambda)$ in this case is referred to [14]).

The other two cases are more interesting but more enigmatic. Indeed, the only examples I am currently aware of are the maps $T^{2} \rightarrow \mathbb{C P}^{1}$ with elliptic spectral curve: these are all Gauss maps of the Delaunay surfaces of constant mean curvature in Euclidean 3-space. Here $\operatorname{dim} J^{\prime}=2$ so the algebraic condition is automatically satisfied.

\section{References.}

[1] E. Arbarello, M. Cornalba, P.A. Griffiths, and J. Harris, Geometry of algebraic curves, Volume I, Springer, New York (1985).

[2] A.I. Bobenko, All constant mean curvature tori in $R^{3}, S^{3}$ and $H^{3}$ in terms of theta-functions, Math. Ann. 290 (1991), 209-245.

[3] F.E. Burstall, D. Ferus, F. Pedit, and U. Pinkall, Harmonic tori in symmetric spaces and commuting Hamiltonian systems, Ann. Math. 138 (1993), 173-212. 
[4] F.E. Burstall and F. Pedit, Harmonic maps via Adler-Kostant-Symes theory, in Harmonic maps and integrable systems, ed: A P Fordy \& J C Wood, Aspects of Mathematics E23, Vieweg 1994.

[5] P. Griffiths and J. Harris, Principles of algebraic geometry, Wiley, New York (1978).

[6] R. Hartshorne, Algebraic geometry, Graduate Texts in Math. 52, Springer, New York (1977).

[7] N.J. Hitchin, Harmonic maps from a 2-torus to the 3-sphere, J. Diff. Geom. 31 (1990), 627-710.

[8] G.R. Jensen and R. Liao, Families of flat minimal tori in $\mathbb{C P}^{n}$, J. Diff. Geom. 42 (1995), 113-132.

[9] K. Kenmotsu, On minimal immersions of $\mathbb{R}^{2}$ into $\mathbb{P}^{n}(\mathbb{C})$, J. Math. Soc. Japan 37 (1985), 665-682.

[10] I. McIntosh, A construction of all non-isotropic harmonic tori in complex projective space, Internat. J. Math. 6 (1995), 831-879.

[11] I. McIntosh, Two remarks on the construction of harmonic tori in $\mathbf{C P}^{n}$, Internat. J. Math. 7 (1996), 515-520.

[12] U. Pinkall and I. Sterling, On the classification of constant mean curvature tori, Ann. Math. 130 (1989), 407-451.

[13] J.-P. Serre, Algebraic groups and class fields, Grad. Texts in Math. 117, Springer, New York (1988).

[14] T. Taniguchi, Non-isotropic harmonic tori in complex projective spaces and configurations of points on Riemann surfaces, Tohoku Mathematical Publ. 14 (1999).

[15] S. Udagawa, Harmonic maps from a two-torus into a complex Grassmann manifold, Internat. J. Math. 6 (1995), 447-459.

[16] K. Uhlenbeck, Harmonic maps into Lie groups (classical solutions of the chiral model), J. Diff. Geom. 30 (1989), 1-50.

[17] J.C. Wood, Holomorphic differentials and classification theorems for harmonic maps and minimal immersions, in Global Riemannian Geometry, 168-175, Eds. T J Willmore \& N J Hitchin, Ellis Horwood, Chichester, 1984. 
UNIVERSITY OF YORK,

HESLINGTON, YORK YO10 5DD,

U.K.

E-mail address: im7@york.ac.uk

RECEIVED July 14, 1999. 